\title{
Le calendrier révolutionnaire de 1793 ou les quatre saisons de la République
}

\author{
The revolutionary calendar of 1793 \\ or four seasons of the Republic
}

\author{
Paweł Matyaszewski \\ Katolicki Uniwersytet Lubelski Jana Pawła II \\ pawelm@kul.pl
}

\begin{abstract}
The authors of the revolutionary calendar, in particular Gilbert Romme and Fabre d'Églantine not only want to put the past behind by implicating a new time and new order but also try to prove the relation between history and nature using the example of the events of the Revolution and their compliance with the laws of the universe. They introduce an innovative nomenclature in order to specify the names of particular days and months but they do not change the natural four-season model of division. The goal of the presented idea is to enrich the natural cycle with a new content expressing the spirit and the objectives of the Republic while following the laws of nature.
\end{abstract}

Keywords: French Revolution, calendar, nomenclature, dechristianization, Fabre d'Églantine, Gilbert Romme

Nul doute que le calendrier républicain de 1793 évoque avant tout, ne fût-ce que par la forme même, la question du temps au sens chronométrique du terme, ce que prouvent autant l'idée majeure de cette entreprise révolutionnaire que l'histoire de ses solutions et modifications successives. L'une et l'autre sont particulièrement révélatrices du projet idéologique ambitieux qu'élaborent les artisans du nouveau calendrier de mesurer le temps différemment, en vue d'effacer le passé, voire de révolutionner le présent ou, mieux encore, pour reprendre ici les termes heureux de Bronisław Baczko, de « décréter l'éternité » (Baczko, 1984). Parmi beaucoup d'autres « emblèmes de la 
Raison » (Starobinski, 1978), la refonte du temps doit constituer un symbole identitaire capital, un signe manifeste de la rupture historique qui se produit en France avec la Révolution de 1789 et, surtout, à partir de la fin de la monarchie.

On sait que, selon l'idée révolutionnaire de remettre le temps à zéro, l'an premier de la nouvelle ère est calculé à partir du 22 septembre 1792, date-césure capitale, le jour de la proclamation de la République. L'insistance des artisans du calendrier, surtout de ses deux auteurs emblématiques, Gilbert Romme et Fabre d'Églantine ${ }^{1}$, sur la date fondatrice de la nouvelle époque dans l'histoire du monde n'a rien d'étonnant, vu qu'elle soutient clairement la logique des événements et leur importance pour la construction de la mythologie révolutionnaire. Or, il est révélateur que le 22 septembre 1792, le jour premier de la liberté et de l'égalité qu'apporte le temps nouveau, soit exactement celui de l'équinoxe d'automne, ce dont s'aperçoivent très vite les révolutionnaires pour y voir directement, de manière enthousiaste, un soutien patent que la Nature offre à l'Histoire, les lois de l'Univers s'accordant parfaitement avec celles de la Révolution. Entre l'avènement de la République et le fait que « le même jour à 9 heures 18 minutes 30 secondes du matin, le soleil est arrivé à l'équinoxe vrai, en entrant dans le signe de la Balance », Gilbert Romme n'hésite pas à découvrir plus qu'une simple coïncidence des événements, afin d'y percevoir ouvertement une interaction cosmologique (Romme, 1793, p. 5).

Mieux encore, que la Nature soutienne la Révolution de manière patente, de sorte que leurs mouvements vont de pair, ou même s'entrelacent mutuellement, il le voit également dans le caractère de la saison de l'année, où la monarchie a été abolie et la république instaurée. Il est capital pour Romme que le commencement de l'ère neuve dans l'histoire de la France tombe exactement sur le début de l'automne. Tout d'abord, il s'agit d'un moment de transition naturel significatif, où l'on passe d'une saison à l'autre, tout comme un glissement similaire s'opère au niveau idéologique, voire historique, quand un système politique succède en faveur de l'autre, à cette différence près que ce changement demeure irréversible et loin d'être cyclique. De plus, la mise en valeur de l'automne devient révélatrice parce que c'est la saison de la récolte, où la terre « prodigue ses dons et paye avec magnificence à l'homme laborieux ses soins, ses fatigues et son industrie» (Romme, 1793, p. 5), par quoi on doit découvrir, une fois de plus, la preuve que le rythme de l'ère révolutionnaire suit de près l'harmonie de la nature. Tout logiquement, voire tout naturellement, le 22 septembre 1792 est le moment attendu de recueillir les produits républicains de la semence du 14 juillet 1789, résultats d'une croissance révolutionnaire foisonnante et irrésistible de plus de trois ans.

\footnotetext{
${ }^{1}$ Romme et Fabre d'Églantine sont autant les auteurs phares du calendrier révolutionnaire que responsables des rapports qu'ils ont présentés, le premier le 20 septembre, le second le 5 octobre 1793, devant la Convention nationale, ce qui par ailleurs a permis à la postérité de leur attribuer l'élaboration de toute l'entreprise. Or, il ne faut pas oublier que la commision chargée de travailler sur le nouveau calendrier comptait aussi d'autres membres éminents, parmi lesquels on doit noter surtout des scientifiques, tels ingénieurs, mathématiciens, astronomes, chimistes, botanistes (voir Shaw, 2011, p. 29-58).
} 
Ainsi donc, si dans son principe fondamental, le nouveau calendrier doit exprimer « la vérité de la nature », pour reprendre ici l'expression de l'autre auteur du projet, Fabre d'Églantine, on le voit très bien dans sa réalisation concrète de 1793 (Fabre d'Églantine, 1793, p. 2). Sans vouloir présenter ici en détail l'histoire de la réforme calendaire (voir Shaw, 2011 ; Perovic, 2012), il faut rappeler que ses artisans rejettent fermement le calendrier grégorien chrétien qui reste en vigueur dans la plupart des pays d'Europe depuis 1582, afin d'élaborer un autre système de mesure et d'organisation du temps (voir Ozouf, 1992a). Ils participent ainsi du mouvement de la déchristianisation qui traverse alors la France (voir Vovelle, 1972 ; Ozouf, 1992b), en proposant une version laïque du calendrier, ce que l'on voit déjà dans leur idée de l'an premier qui doit faire débuter et, en même temps, dater l'histoire du monde autrement que par rapport à la religion chrétienne. Néanmoins, en dépit de leur ambition de découper et de séculariser le temps, ils demeurent pourtant fidèles à l'idée de calendrier solaire, par ailleurs la forme la plus fréquente dans l'histoire de l'humanité, en quoi il faut voir, entre autres, leur respect des lois et des cycles de la nature. Ainsi préservent-ils la division mensuelle de l'année et gardent le nombre des douze mois, à ceci près que, selon leur système décimal, chaque mois est divisé, tout comme par exemple dans le calendrier égyptien antique, en trois décades égales et compte exactement 30 jours, ce à quoi on aura encore l'occasion de revenir plus bas. Par conséquent, ils conservent aussi l'idée des quatre saisons de l'année, chacune composée de trois mois consécutifs qui assurent leurs caractéristiques météorologiques distinctives et, surtout, soulignent leur alternance naturelle. La différence fondamentale par rapport au calendrier grégorien réside pourtant dans le fait que, suite à la date fondatrice du 22 septembre - le premier jour de l'an I, donc le début tant de l'ère nouvelle que de l'année - le commencement de cette dernière devra, par conséquent, tomber à chaque fois sur l'avènement de l'automne. De plus, et cela constitue l'un des signes les plus flagrants de la réforme calendaire de 1793, les noms des douze mois des quatre saisons de l'année ont à exprimer un système de valeurs neuf, voire promouvoir l'esprit de la République, donc il faut à tout prix en proposer une nouvelle nomenclature.

Celle-ci, contrairement à l'ancienne, « monument de servitude et d'ignorance », rejette le passé et privilégie le présent en vue d'accentuer la discontinuité du temps, car elle « n'est ni céleste, ni mystérieuse ; elle est toute puisée dans notre révolution » (Romme, 1793, p. 10). Dans la recherche d'une nomenclature neuve, Gilbert Romme, mathématicien et homme politique, propose un critère essentiellement idéologique pour désigner les mois, en faisant à chaque fois allusion à la Révolution « dont elle présente ou les principaux événements, ou le but, ou les moyens » (Romme, 1793, p. 10). Il développe une suite lexicale de nouveaux noms des mois que l'on doit lire comme une histoire abrégée de la Révolution présentée en douze scènes successives : République, Unité, Fraternité, Liberté, Justice, Égalité, Régénération, Réunion, Jeu de Paume, Bastille, Peuple, Montagne. Bronisław Baczko a tout à fait raison d'y voir 
« un temps-récit », où l'auteur tient à raconter la révolution ou, mieux encore, à répéter dans un cycle annuel ses événements et principes fondamentaux, de sorte que chaque année suivante devra les reprendre et réaffirmer (Baczko, 1978, p. 222). Il est vrai que chez Romme la narration itérative d'une révolution condensée en un an est à la fois historique et idéologique : il ne rappelle que la Révolution en France, aussi bien son déroulement que ses idéaux, en privilégiant ainsi le national à l'universel et, paradoxalement, en négligeant la Nature en faveur de l'Histoire. Curieusement, dans un projet calendaire qui veut inscrire les événements révolutionnaires dans la logique inébranlable et sacrée des lois de l'Univers, son auteur ne fait qu'une seule fois allusion à la nature et à une saison de l'année : « le mois de la Régénération est le premier du printemps, où toute la nature se régénère » (Romme, 1793, p. 12).

Chargé, lui aussi, de proposer une nouvelle nomenclature du calendrier révolutionnaire, Fabre d'Églantine, poète et dramaturge, donne peu de temps après Romme une version que la Convention acceptera avec enthousiasme le 24 octobre 1793 et qui, aujourd'hui encore, fait évoquer, selon Mona Ozouf, « la tendresse nostalgique qu'on montre parfois aux beaux noms imaginés » et y voir « le génie bucolique de la sensibilité révolutionnaire» (Ozouf, 1992a, p. 92). À vrai dire, tout comme son prédécesseur, Fabre d'Églantine présentera un véritable calendrier-narration, à cette différence près que son récit devra puiser essentiellement, sinon uniquement, dans le monde de la nature et de la vie de campagne, en vue « de consacrer par le calendrier le système agricole et d'y ramener la nation, en marquant les époques et les fractions de l'année par des signes intelligibles ou visibles pris dans l'agriculture ou l'économie rurale » (Fabre d'Églantine, 1793, p. 6). On comprend que, par opposition au récit événementiel élaboré par Romme, sa proposition tienne non seulement à être poétique, voire plus originale et plus belle au niveau lexical, mais aussi, sinon surtout, à devenir universelle, entièrement ancrée dans le temps de la nature. Le calendrier de l'Histoire cède décidément la place à celui de la Nature, l'universel l'emportant clairement sur le national.

Ainsi donc, dans sa tendance « à frapper l'imagination par les dénominations et à instruire par la nature et la série des images » (Fabre d'Églantine, 1793, p. 6), Fabre d'Églantine montre avant tout un talent de poète sensible aux subtilités de la langue et invente des néologismes qui puissent exprimer et faire mémoriser la « vérité de la nature ». Sans chercher à construire des dénominations spéciales pour désigner les quatre saisons de l'année, l'auteur exprime pourtant très bien leurs traits caractéristiques lorsqu'il propose des sonorités évocatrices distinctes et distinctives, « de telles manières que les noms des mois qui composent l'automne ont un son grave et une mesure moyenne ; ceux de l'hiver un son lourd et une mesure longue ; ceux du printemps un son gai et une mesure brève ; et ceux de l'été un son sonore et une mesure large » (Fabre d'Églantine, 1793, p. 6). Les désinences ternaires s'appuient donc sur des distinctions à la fois climatiques et linguistiques, l'ensemble étant assuré par une musicalité et une rythmique poétiques irremplaçables. Paradoxalement, et là réside le sens de la proposition de Fabre d'Églantine, les quatre saisons de l'année n'ont pas 
besoin de dénominations spéciales pour être facilement retrouvées dans le calendrier grâce à la même sonorité des triades mensuelles qui les composent successivement. Dans son idée de "donner à chacun des mois de l'année un nom caractéristique ", il s'agit avant tout de faire « sentir le genre de saison, où il se trouve dans les quatre dont se compose l'année » (Fabre d'Églantine, 1793, p. 6).

À l'intérieur de chaque saison, l'auteur répertorie et met en valeur, en vrai météorologue, les traits particuliers des douze mois de l'année, voire le contenu du temps, à partir duquel il invente ses néologismes, où le climat et la langue s'enchaînent mutuellement et offrent des propositions poétiques originales. Dans l'automne, il insiste visiblement sur trois traits saisonniers distinctifs : la récolte des fruits de la terre (Vendémiaire, du 22 septembre au 21 octobre, selon l'ancien calendrier grégorien), l'état dense de l'atmosphère (Brumaire, du 22 octobre au 20 novembre), la température basse de l'air (Frimaire, du 21 novembre au 20 décembre), le tout lié par un suffixe adjectival commun « aire » venant de la terminaison latine « arius » désignant à la fois «l'appartenance » et « l'origine ». La narration du calendrier-récit est ici bien claire : en automne, tout comme l'a signalé auparavant Gilbert Romme, l'homme termine ses travaux champêtres et recueille les produits que lui procure la nature qui, de son côté, sous les coups des brumes et des brouillards, change de caractère et, surtout, de température, et prépare déjà au froid de l'hiver.

Quant à celui-ci, Fabre d'Églantine tient à distinguer dans l'hiver trois états de l'atmosphère différents : l'eau congelée et glaciale qui couvre la terre (Nivôse, du 21 décembre au 19 janvier), l'eau sous forme liquide tombant du ciel en grande quantité (Pluviôse, du 20 janvier au 18 février), l'air en mouvement, tantôt sec, tantôt pourvu d'averses (Ventôse, du 19 février au 20 mars). Les trois mois hivernaux sont mis au même rang lexical par la terminaison commune " ôse » venant du suffixe latin « osus », c'est-à-dire « doté de », qui renforce encore mieux l'idée d'abondance. Cette fois-ci, la narration emprunte essentiellement aux conjonctures météorologiques de la nature : de la période froide de la neige, on passe par le temps humide des pluies, pour entrer dans l'air desséchant des vents qui disposent la terre au printemps.

L'emblème fondamental de la nomenclature que propose Fabre d'Églantine pour ce dernier est le sol dans ses trois étapes de croissance successives : la germination (Germinal, du 21 mars au 19 avril), la floraison (Floréal, du 20 avril au 19 mai), l'épanouissement des prairies (Prairial, du 20 mai au 18 juin). La terminaison « al » commune aux trois mois printaniers vient du suffixe adjectival latin « alis » signifiant, tout comme « arius », l'idée d'appartenance et d'origine, afin de soutenir l'unité du message biologique de cette partie du calendrier qui, de son côté, poursuit toujours la logique de la narration. Au printemps la nature prépare la terre à ressusciter et à reprendre sa richesse de vie ; elle la fait se développer à neuf, donner naissance aux grains, pousser des plantes et des fleurs.

La référence à la récolte des prairies au dernier mois printanier permet à l'auteur de passer habilement à la saison de l'été qui rappelle les travaux champêtres de la 
récolte des blés (Messidor, du 19 juin au 18 juillet), la température estivale élevée (Thermidor, du 19 juillet au 17 août), l'abondance et la saveur des fruits (Fructidor, du 18 août au 16 septembre). Que derrière ces désinences se cache l'idée d'opulence bienveillante de la nature, l'auteur l'exprime très bien par la terminaison commune « idor » empruntée au terme grec « dôron », ce qui veut dire « don ». De cette manière, le récit de l'été représente l'image d'une nature généreuse qui, en signe de récompense pour les efforts assidus de l'homme, exécutés dans une période de chaleur difficile, lui offre abondamment les riches produits de la terre.

Ainsi donc, tout comme dans le récit événementiel historique de Romme, le cycle narratif du calendrier révolutionnaire de Fabre d'Églantine forme une entité de mois et de saisons cohérente, où la répétition des phénomènes climatiques et ruraux tient à réaffirmer la vérité du temps de la nature. Mieux encore, chez lui cette reprise constante du cycle annuel se voit encore plus prononcée par un lien contextuel évident entre le dernier des mois de la dernière saison et le premier des mois de la première saison de l'année. Fructidor, mois du mûrissement des fruits, semble tout logiquement annoncer Vendémiaire, mois de la récolte des raisins, par quoi on reçoit à la fois l'idée de passage et celle de la continuité. De cette manière, la fin du dernier mois impose pour ainsi dire le début du premier, l'achèvement de la dernière saison exige l'avènement de l'autre, l'ensemble du cycle annuel se refermant ainsi tout naturellement, afin de se réouvrir ensuite pour toute l'année suivante ${ }^{2}$.

Bien entendu, la nomenclature qu'élabore Fabre d'Églantine ne serait ni cohérente, ni complète sans proposer en même temps les dénominations des 360 jours qui meublent les douze mois et les quatre saisons de l'année du calendrier révolutionnaire. Par opposition à la religion chrétienne, aux saints et patrons du calendrier grégorien, " les squelettes béatisés tirés des catacombes de Rome », il introduit un catalogue laïc de " tous les objets qui composent la véritable richesse nationale » et dont les exemples doivent embrasser l'ensemble du travail agricole et de la vie rurale (Fabre d'Églantine, 1793, p. 12). Il les classifie en trois groupes principaux distincts, tous liés étroitement au contexte de la nature : « les utiles productions de la terre, les instruments dont nous nous servons pour la cultiver, et les animaux domestiques, nos fidèles serviteurs dans ces travaux » (Fabre d'Églantine, 1793, p. 12). Contrairement aux désinences des mois, l'auteur n'a plus besoin d'inventer des néologismes poétisants pour nommer les jours consécutifs de l'année révolutionnaire, mais recourt à un vocabulaire commun de la langue, connu et acquis de tous, pour y puiser des termes empruntant directement à l'univers de la campagne et de l'agriculture.

${ }^{2}$ Il est vrai qu'entre le 30 Fructidor et le $1^{\text {er }}$ Vendémiaire, le calendrier républicain propose une suite de « sans-culottides », série de fêtes révolutionnaires consécutives qui ont à « combler» le nombre des jours de l'année solaire pour suivre le rythme de la nature. Il s'agit d'épagomènes, cinq jours complémentaires chaque année commune et six jours toutes les années bissextiles. L'idée des « sans-culottides », situés entre la fin et le début de chaque année, doit encore mieux accentuer l'accord de la Révolution avec la Nature et prouver leur interpénétration inaltérable. 
Or, il ne s'agit pourtant pas d'une simple énumération désordonnée des trois composants lexicaux de la nomenclature journalière. Au contraire, l'auteur tient à les inscrire habilement dans la logique de son calendrier-récit où, après l'histoire des cycles de la nature racontée à travers les mois et les saisons de l'année, la narration doit continuer et reprendre sa suite, à commencer par le premier groupe de jours distingué, " les grains, les patûrages, les arbres, les racines, les fleurs, les fruits, les plantes » (Fabre d'Églantine, 1793, p. 12) ${ }^{3}$. L'apparition de cette catégorie des éléments calendaires qui doivent désigner les jours, la plus vaste car composée de 288 noms, a sans aucun doute pour sa fonction fondamentale de montrer la richesse de la nature et de prouver la prospérité de la vie rurale. Néanmoins, l'ordre de la répartition des noms des « utiles productions de la terre » suit entièrement « le temps et le jour où la nature nous en fait présent » (Fabre d'Églantine, 1793, p. 12) et respecte le caractère des mois et des saisons qui la composent successivement, de sorte que les dénominations des jours complètent et confirment tout logiquement la vérité de toute l'année.

Il en est de même avec les noms des animaux qui apparaissent dans le calendrier. Il s'agit surtout d'animaux domestiques, mammifères, oiseaux, insectes ou poissons, qui aident l'homme dans ses travaux de campagne, le nourrissent, ou tout simplement rendent son travail plus agréable par leur chant et leur compagnie. Il faut rappeler ici que, selon le projet de Fabre d’Églantine, chaque mois est divisé en trois décades, chacune composée de dix jours, appelés successivement : primedi, duodi, tridi, quartidi, quintidi, sextidi, septidi, octidi, nonidi, décadi. La répartition des noms des animaux à chaque quintidi, voire à chaque demi décade, les 5, 15 et 25 de chaque mois, n'est pas l'effet du hasard, car l'auteur tient par là à établir un « rapport précis entre la date de cette inscription et l'utilité réelle de l'animal inscrit " (Fabre d'Églantine, 1793, p. 13). Le catalogue de 36 animaux répertoriés, de même que l'ordre de leur apparition dans le calendrier, doivent donc autant montrer leur présence dans l'environnement rural de l'homme, que mettre en valeur la vérité selon laquelle celle-ci survient selon le temps de la nature, en respectant les mois et les saisons de l'année.

Fabre d'Églantine reste également fidèle à sa logique inébranlable dans la manière de systématiser les outils ou appareils de campagne, car « chaque décadi est marqué par le nom d'un instrument aratoire, le même dont l'agriculteur se sert au temps précis où il est placé » (Fabre d'Églantine, 1793, p. 13). Là aussi, le catalogue de 36 noms des ustensiles agricoles semble conforme à la saison de l'année et soumis à leur utilité selon un mois de travail. Dans son souci du narrateur, l'auteur veut même donner une suite logique à leur apparition à l'intérieur de chaque mois, en appropriant leur fonction au caractère des travaux et aux besoins de l'agriculture suivant les décades consécutives. Mieux encore, la disposition d'un outil à chaque décadi est l'effet d'une

${ }^{3}$ Conscient que « la terre est scellée et communément couverte de neige » au mois de Nivôse, l'auteur propose pour ce « temps du repos de la terre [...] les productions, les substances du règne animal ou minéral, immédiatement utiles à l'agriculture » (Fabre d'Églantine, 1793, p. 13-14). 
mise en scène voulue de l'auteur. Il faut rappeler que le décadi est l'équivalent laïc du dimanche, terme disparu dans le calendrier révolutionnaire, mais dont la nouvelle nomenclature garde le caractère du jour de fête et de repos. Le fait que chaque décadi soit marqué par la présence d'un instrument aratoire n'est pas fortuit, mais s'inscrit dans la logique du récit du calendrier, car « le laboureur dans le jour de repos retrouvera consacré, dans le calendrier, l'instrument qu'il doit reprendre le lendemain » (Fabre d'Églantine, 1793, p. 13). Ainsi donc, le jour chômé est en même temps celui de l'instruction qui rappelle à l'agriculteur les devoirs qui l'attendent au début de la décade suivante et lui indique le sens de ses travaux et efforts à venir.

Ce caractère éducatif est par ailleurs le signe le plus emblématique de tout le calendrier républicain. En effet, celui-ci est fait, selon Fabre d'Églantine, « pour glisser parmi le peuple les notions rurales élémentaires, pour lui montrer la richesse de la nature, pour lui faire aimer les champs, et lui désigner avec méthode, l'ordre des influences du ciel et des productions de la terre » (Fabre d'Églantine, 1793, p. 12). Il s'agit d'un véritable almanach agricole qui ne cesse pourtant de garder sa fonction majeure du calendrier-narration. Par là, à côté de sa dimension idéologique, le calendrier de 1793 est doté d'un rôle à la fois philosophique et pragmatique, en rappelant ainsi l'entreprise encyclopédique de Diderot. Il offre à vrai dire la version révolutionnaire d'une encyclopédie en poche, du moins en ce qui concerne le domaine de la vie rurale et de l'agriculture. Il est à remarquer que cette disposition est possible surtout grâce à son sens pictural profond. Convaincu que « nous ne concevons rien que par des images [...], notre entendement ne se rend compte que par des images, notre mémoire ne s'appuie et ne se repose que sur des images » (Fabre d'Églantine, 1793, p. 2), Fabre d'Églantine insiste très fort sur la dimension iconographique du calendrier. Il tient à faire travailler l'imaginaire à l'aide de la nomenclature qu'il invente et des représentations qu'il propose, au point de supposer que « à chaque instant de l'année, du mois, de la décade et du jour, les regards et la pensée du citoyen se porteront sur une image agricole, sur un bienfait de la nature, sur un objet d'économie rurale » (Fabre d'Églantine, 1793, p. 2-3).

Des artistes français ne tarderont pas à se rendre compte du potentiel plastique des « images parlantes » du calendrier révolutionnaire et de sentir ses possibilités esthétiques capitales. La réalisation la plus célèbre est celle du peintre Salvatore Tresca qui, sous le Directoire, entre 1797-1798, se charge d'exécuter, d'après les dessins de 1796 d'un autre artiste, Louis Lafitte, des planches colorées illustrant le calendrier républicain. Faisant inévitablement penser à l'initiative de l'Encyclopédie d'accompagner l'écrit par une image-commentaire explicative et embellissante, les douze planches illustrées représentent, sous forme d'allégories féminines, les nouveaux mois de l'année, en gardant, de manière visible, leurs désinences ternaires distinctes, propres aux quatre saisons successives. Les images traduisent ainsi en langage de la peinture la beauté et la sensibilité de la nomenclature du calendrier révolutionnaire, de même qu'elles expriment parfaitement le message du nouveau temps républicain. 


\section{BIBLIOGRAPHIE}

Baczko, B. (1978). Lumières de l'utopie. Paris : Payot.

Baczko, B. (1984). Le calendrier républicain : décréter l'éternité. In P. Nora (Ed.), Les lieux de mémoire. Vol. I : La République (pp. 37-83). Paris : Gallimard.

Fabre d'Églantine, Ph. (1793). Rapport fait à la Convention Nationale dans la séance du 3 de second mois de l'an II de la République au nom de la commission chargée de la confection du calendrier. Paris : Imprimeries Nationales. Retrieved from https://gallica.bnf.fr/ark:/12148/bpt6k48746z.image.

Ozouf, M. (1992a). Calendrier. In F. Furet, M. Ozouf (Eds.), Dictionnaire critique de la Révolution française. Vol. III : Institutions et créations (pp. 91-106). Paris : Flammarion.

Ozouf, M. (1992b). Déchristianisation. In F. Furet, M. Ozouf (Eds.), Dictionnaire critique de la Révolution française. Vol. I : Événements (pp. 79-99). Paris : Flammarion.

Perovic, S. (2012). The Calendar in Revolutionary France. Perceptions of Time in Literature, Culture and Politics. Cambridge: University Press. DOI: org/10.1017/CBO9781139198950.

Romme, G. (1793). Rapport sur l'ère de la République fait à la Convention nationale dans la séance du 20 septembre de l'an II de la République. Paris : Imprimeries Nationales. Retrieved from https://gallica.bnf.fr/ark:/12148/bpt6k487479/f12.image.

Shaw, M. (2011). Time and the French Revolution: The Republican Calendar, 1789-XIV Year. London : Royal Historical Society, Boydel Press.

Starobinski, J. (1973). 1789 - Les Emblèmes de la Raison. Paris : Flammarion.

Vovelle, M. (1976). Révolution et religion. La déchristianisation de l'an II. Paris : Hachette. 